\title{
OR13-004 - Evidence-based clinical classification criteria for periodic fevers
}

\author{
S Federici ', S Ozen, I Koné-Paut, H Lachmann, P Woo, L Cantarini, G Amaryan, A Insalaco, J Kuemmerle-Deschner, \\ B Neven, N Dewarrat, Y Uziel, D Rigante, T Herlin, S Martino, A Simon, S Stojanov, H Ozdogan, J Frenkel, \\ N Ruperto, A Martini, M Sormani, M Hofer, M Gattorno
}

From 7th Congress of International Society of Systemic Auto-Inflammatory Diseases (ISSAID) Lausanne, Switerland. 22-26 May 2013

\section{Introduction}

No evidence-based classification criteria are so far available the majority of autoinflammatory diseases

\section{Objectives}

To elaborate and validate a set of clinical criteria able to correctly classify patients affected with the most common periodic fevers.

\section{Methods}

All FMF, TRAPS, MKD and CAPS patients enrolled in the Eurofever registry until March 2013 were evaluated. For each disease gold standards were considered according to the following criteria: i) clinical validation by centers and disease-principal investigator, ii) confirmative molecular analysis (2 mutations for MEFV with at least one mutation in exon 10, 2 mutations of MVK gene, 1 mutation of TNFRFS1A with exclusion of low-penetrance variants, 1 mutation of NLRP3 with exclusion of low-penetrance variants), iii) PFAPA patients validated by disease-principal investigator and confirmed by the centers on the basis of the follow-up. Clinical criteria was formulated on the basis of a univariate and multivariate analysis in a first group of patients (training set) and validated in an independent set of patients (validation set).

\section{Results}

A total of 1204 consecutive patients with periodic fevers were enrolled in the registry. Among them 743 consecutive gold standard patients (288 FMF, 73 MKD, 96 TRAPS, 87 CAPS, 199 PFAPA) were evaluated (440 in the training set and 303 in the validation set). The multivariate analysis identified the clinical variables (either as presence or absence).

\footnotetext{
GASLINI, Genova, Italy
}

C 2013 Federici et al; licensee BioMed Central Ltd. This is an Open Access article distributed under the terms of the Creative Commons Attribution License (http://creativecommons.org/licenses/by/2.0), which permits unrestricted use, distribution, and reproduction in any medium, provided the original work is properly cited.
The classification score was than tested in an independent set of patients (validation set) revealing a sensitivity of $93 \%$ and specificity of $89 \%$ for FMF; a sensitivity of $100 \%$ and specificity of $74 \%$ for TRAPS; a sensitivity of $80 \%$ and specificity of $90 \%$ for MKD and sensitivity of $97 \%$ and specificity of $92 \%$ for CAPS; sensitivity of $99 \%$ and specificity of $96 \%$ for PFAPA. The performance in non-gold standard patients (i.e. heterozygous patients in autosomal recessive diseases or patients with low-penetrance mutations) revealed a variable percentage of patients (70\% FMF, 75\% TRAPS, $41 \%$ MKD and 94\% CAPS) positive for the respective criteria.

Independently correlated to for each disease with their specific weight. The cut off value of the classification score was chosen on the ROC curve in order to guarantee the highest sensitivity and specificity.

\section{Conclusion}

Evidence-based clinical criteria for the classification of patients with inherited periodic fevers have been elaborated. These clinical criteria could be used in association with molecular analysis and other variables (i.e. metabolic examinations, response to specific treatments) for patients classification.

\section{Disclosure of interest \\ None declared.}

Published: 8 November 2013

doi:10.1186/1546-0096-11-S1-A266

Cite this article as: Federici et al.: OR13-004 - Evidence-based clinical

classification criteria for periodic fevers. Pediatric Rheumatology 2013 11(Suppl 1):A266. 